\title{
Inertia Effects in the Flow of a Herschel-Bulkley ERF between Fixed Surfaces of Revolution
}

\author{
A. Walicka and J. Falicki \\ University of Zielona Góra, Department of Mechanics and Design of Machines, ul. Szafrana 2, P.O. Box 47, \\ 65-516 Zielona Góra, Poland
}

Correspondence should be addressed to A. Walicka; a.walicka@ijame.uz.zgora.pl

Received 28 March 2013; Accepted 13 June 2013

Academic Editor: Hideki Hosoda

Copyright (C) 2013 A. Walicka and J. Falicki. This is an open access article distributed under the Creative Commons Attribution License, which permits unrestricted use, distribution, and reproduction in any medium, provided the original work is properly cited.

Many electrorheological fluids (ERFs) as fluids with microstructure demonstrate viscoplastic behaviours. Rheometric measurements indicate that some flows of these fluids may be modelled as the flows of a Herschel-Bulkley fluid. In this paper, the flow of a Herschel-Bulkley ER fluid-with a fractional power-law exponent-in a narrow clearance between two fixed surfaces of revolution with common axis of symmetry is considered. The flow is externally pressurized, and it is considered with inertia effect. In order to solve this problem, the boundary layer equations are used. The influence of inertia forces on the pressure distribution is examined by using the method of averaged inertia terms of the momentum equation. Numerical examples of externally pressurized ERFs flows in the clearance between parallel disks and concentric spherical surfaces are presented.

\section{Introduction}

In recent years, the study of fluids with microstructures has gained much importance because of its numerous applications in various engineering disciplines such as chemical engineering, polymer processing, plastic forming foundry engineering, and engineering of lubrication [1-14].

In machines and mechanisms systems of many industrial processes, the phenomena of a flow of viscoplastic fluids are used. One of these phenomena is a slide bearing lubrication $[9,10,13]$. Advances in technology and severe operational requirements of machines necessitated the development of improved lubricants to ensure a smooth and safe operation. Generally, viscosity of lubricants decreases with temperature. For operations under high speeds and heavy loads, oils containing high molecular weight polymers as viscosity index improvers are used to increase a load carrying capacity of the modified lubricants $[9,13]$.

Most substances used in the lubrication technology are polymer solutions, thus, the characteristics of the bearings change when such rheological substances, known as nonNewtonian fluids, are used as lubricants. Several constitutive relations applied were used to model the non-Newtonian characteristics exhibited by some lubricants $[7,11,13,15,16]$.

Another ones of these phenomena are processes of vibration control and torque transmission. In the last years, the electrorheological fluids (abbreviated to ERFs) have acquired a great relevance for supporting vibration control and torque transmission devices, based on the characteristic dependence of their viscosity on applied electric field strength. Since their initial discovery by Winslow [17], many particle-dispersion electrorheological fluids, consisting of dielectric particles dispersed in insulating oil, have been reported. When the external electric field is imposed to an ERF, it behaves as a viscoplastic fluids $[9,18-25]$, displaying a field-dependent yield shear stress which is widely variable. Without the electric field, the ERF has a reversible and a constant viscosity so that it flows as a Newtonian fluid. Another salient feature of the ERF is that the time required for the viscosity variation is very short $(<0.001 \mathrm{sec})$. These attractive characteristics of the ERF provide the possibility of the appearance of new engineering technology. Recently, the application of the ERF to rotor-bearing systems has also been initiated by Basavaraja et al. [2], Jung and Choi [18], Dimarogonas and Kollias [26], 
and Peng and Zhu [27] and vibration control by Lee and Wereley [23], Choi et al. [24], El Wahed et al. [28], and Lim et al. [29].

To describe the rheological behaviour of viscoplastic fluids in complex geometries, the Bingham model is used $[8,13,15,16,21]$. Recently, the nonlinear model of Shulman et al. [21] has been successfully applied $[8,13,15,16,20]$. The constitutive equation for this model is given as follows:

$$
\tau=\left[\tau_{0}^{1 / n}+(\mu \dot{\gamma})^{1 / m}\right]^{n}
$$

where $\tau$ is the shear stress, $\tau_{0}$ is the yield shear stress, $\mu$ is the coefficient of plastic viscosity, $\dot{\gamma}$ is the shear strain rate, and $m$ and $n$ are the power-law exponents. By reducing the coefficients in the Shulman equation one can obtain simpler models describing the flow of a viscoplastic fluid.

The most popular model of the ERF is the Bingham model for which $m=n=1$ in (1). Recently, the nonlinear model of Herschel-Bulkley has been successfully used to describe the viscoplastic behaviour of nonNewtonian ERFs. The constitutive equation for this model is as follows $[5,30$, 31]:

$$
\tau=\tau_{0}+(\mu \dot{\gamma})^{1 / m} .
$$

The purpose of this paper is a study of pressure distribution in a flow of the Herschel-Bulkley ERF-with a fractional power-law exponent [8] - in the clearance modelled as a narrow space between two surfaces of revolution shown in Figure 1. Using the method of averaged inertia [32] (similar method is used in [33]), the influence of inertia terms of the equations of motion and viscoplastic behaviour on this distribution is analysed. To solve the problem we will use the lubrication approximation theory to the flow in a narrow clearance $[8,10,15,16,25]$.

\section{Equations of Motion of the ERF}

The yield shear stress for the ERF varies with respect to the electric field. According to the experimental results reported in Shulman et al. [21], Shulman and Nosov [22], Jordan and Shaw [34], and Otsubo [35], the relation between the yield shear stress $\tau_{0}$ and the electric field strength $\mathbf{E}$ is given as follows:

$$
\tau_{0}(E)=\alpha\left(\frac{U}{2 h}\right)^{\beta},
$$

where $E$ is the magnitude of the vector $\mathbf{E}$ but $U$ and $2 h$ are the applied voltage and the film thickness, respectively. Both parameters $\alpha$ and $\beta$ are the experimental constants of which the range of the exponent $\beta$ is 1 to 2.4 (Jordan and Shaw [34], Otsubo [35]). Other experimental data (Whittle et al. [36]) suggest that the yield shear stress for some ERFs is as follows:

$$
\tau_{0}(E)=\alpha_{1}\left(\frac{U}{2 h}\right)+\alpha_{2}\left(\frac{U}{2 h}\right)^{2},
$$

where $\alpha_{1}$ and $\alpha_{2}$ are constants; for relatively high field strengths a simpler formula may be used

$$
\tau_{0}(E)=\alpha\left(\frac{U}{2 h}\right)^{2},
$$

where $\alpha$ is an experimental constant.
The three-dimensional constitutive equation for the Herschel-Bulkley ERF has the form $[15,16,25]$ :

$$
\begin{gathered}
\mathbf{T}=-p \mathbf{1}+M \mathbf{A}_{1}, \quad M=\left[\tau_{0}+(\mu A)^{1 / m}\right] A^{-1}, \\
A=\left[\frac{1}{2} \operatorname{tr}\left(\mathbf{A}_{1}^{2}\right)\right]^{1 / 2},
\end{gathered}
$$

where $\mathbf{1}$ is the unit tensor, $p$ is the pressure, $A$ is second invariant of the stretching tensor $\mathbf{A}_{1}$ (the first Rivlin-Ericksen tensor) defined by

$$
\mathbf{A}_{1}=\mathbf{L}+\mathbf{L}^{T}, \quad \mathbf{L}=\operatorname{grad} \mathbf{v},
$$

and $\mathbf{v}$ is velocity vector.

The general equations of motion of the Herschel-Bulkley fluid have a form:

(i) equation of continuity:

$$
\operatorname{div} \mathbf{v}=0
$$

(ii) equation of momentum

$$
\rho \frac{d \mathbf{v}}{d t}=\operatorname{div} \mathbf{T}
$$

or

$$
\rho \frac{d \mathbf{v}}{d t}=-\nabla p+\operatorname{div} \boldsymbol{\Lambda}
$$

where

$$
\Lambda=M \mathbf{A}_{1}
$$

here $M$ is a coefficient of viscoplasticity.

Let us consider the Herschel-Bulkley ERF in a clearance between fixed surfaces of revolution. The flow configuration is shown in Figure 1. The surfaces of revolution are defined by function $R(x)$ which denotes the radius of the median between the surfaces, plus function $h(x)$ which denotes the distance to each surfaces from the median, measured along a normal to the median. An intrinsic curvilinear orthogonal coordinate system $x, \vartheta, y$ is also depicted in Figure 1 .

The physical parameters of the lubricant flow are the velocity components $v_{x}, v_{y}$, and pressure $p$. With regard to the axial symmetry of the flow these parameters are not dependent on the angle $\vartheta$.

The assumption typical for the flow in a narrow clearance $[2,8,15,16]$

$$
h(x) \ll R(x),
$$

can be used to make order-of-magnitude arguments for $(8) \div$ (11).

A further simplification comes by noting that-in accordance to the lubrication approximation-the most important changes in an annular channel occur in the normal (to the channel median) direction. This leads to the assumption 


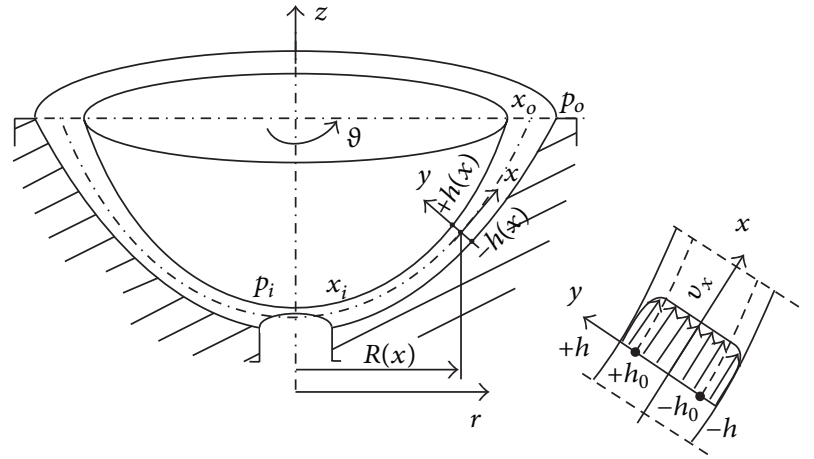

FIGURE 1: The geometry of a curvilinear clearance between two surfaces of revolution.

that the flow is nearly parallel to the surfaces bounding the clearance, so that

$$
v_{y} \ll v_{x}, \quad \frac{\partial}{\partial x} \ll \frac{\partial}{\partial y},
$$

in an intrinsic coordinate system.

If some asymptotic transformations are made, the same as in (Falicki [8], Walicka $[15,16]$ ), these equations can be reduced to a simpler form:

$$
\begin{gathered}
\frac{1}{R} \frac{\partial\left(R v_{x}\right)}{\partial x}+\frac{\partial v_{y}}{\partial y}=0 \\
\rho\left(v_{x} \frac{\partial v_{x}}{\partial x}+v_{y} \frac{\partial v_{x}}{\partial y}\right)=-\frac{d p}{d x}+\frac{\partial T_{y x}}{\partial y}
\end{gathered}
$$

where

$$
\begin{gathered}
T_{y x}=S\left[\tau_{0}+\left(\mu\left|\frac{\partial v_{x}}{\partial y}\right|\right)^{1 / m}\right], \\
\tau_{0}(x)=\alpha\left[\frac{U(x)}{2 h(x)}\right]^{\beta},
\end{gathered}
$$

and $S=\operatorname{sgn}\left(d v_{x} / d y\right)$. The signum (sgn) function takes on the value +1 for a positive argument and -1 for a negative argument.

The order-of-magnitude arguments show that

$$
p=p(x)
$$

is a function of $x$ only.

For a majority of greases, molten polymers, mush metals, and ERFs, the values of a yield shear stress are contained in the limits: $0(1) \leq \tau_{0} \leq 0(3)$, where $0(\alpha) \sim 10^{\alpha}$ and denotes the order of magnitude, but the exponent $m$ is equal to: $m=$ $4 / 3$ or $m=2.5$, and it is not an integer number (Falicki [8], Roussel [12]).

In the flow of a fluid with the yield shear stress, there exists a quasi-solid core flow bounded by surfaces laying at

$$
\begin{gathered}
|y|=h_{0} \text { for which the shear stresses are } \\
\qquad\left|T_{y x}\right|=\tau_{0} .
\end{gathered}
$$

Combining the expressions (16) and (18), one obtains the boundary conditions on liquid surfaces of the core flow as

$$
\frac{\partial v_{x}}{\partial y}=0 \text { for } y= \pm h_{0}
$$

The boundary conditions on solid surfaces are stated as follows:

$$
v_{x}=v_{y}=0 \quad \text { for } y= \pm h
$$

On the median line there is also

$$
\frac{\partial v_{x}}{\partial y}=0 \text { for } y=0 \text { and hence }\left|T_{y x}\right|=0 \text { for } y=0 \text {. }
$$

Moreover, in the inlet and the outlet of the clearance conditions for the pressure can be written in the form

$$
p\left(x_{i}\right)=p_{i}, \quad p\left(x_{o}\right)=p_{o}
$$

where $x_{i}$ denotes the inlet coordinate and $x_{o}$-outlet coordinate.

\section{Solution to the Equation of Motion}

Taking into account (14), one can rearrange (15) writing it in the form

$$
\begin{aligned}
& \rho\left(\frac{1}{R} \frac{\partial\left(R v_{x}^{2}\right)}{\partial x}+\frac{\partial}{\partial y}\left(v_{x} v_{y}\right)\right) \\
& \quad=-\frac{d p}{d x}+\frac{\partial}{\partial y}\left(S\left[\tau_{0}+\left(\mu\left|\frac{\partial v_{x}}{\partial y}\right|\right)^{1 / m}\right]\right) .
\end{aligned}
$$

Then, averaging the left-hand side of (23) across the clearance thickness, solving the obtained equation and taking into account the boundary conditions (20)-(22) we get the following result for the pressure distribution $[8,15,16,37-39]$ :

$$
\begin{gathered}
p=p_{o}+\left[S(x)-S_{o}\right]+\left[D(x)-D_{o}\right], \\
S_{o}=S\left[x_{o}\right], \quad D_{o}=D\left[x_{o}\right] .
\end{gathered}
$$

Functions $S(x)$ and $D(x)$ depend upon the solution $X_{s}$ of the equation characteristic to the flow of a Herschel-Bulkley fluid in the clearance; this equation has a form $[8,16,37,39]$ :

$$
\frac{(X-1)^{m+1}}{(m+1)(m+2)}[X(m+1)+1]-K X^{2}=0,
$$

where

$$
K=\frac{\mu Q}{4 \pi R h^{2} \tau_{0}^{m}}, \quad X=\frac{h}{h_{0}},
$$

and $Q=2 \pi R \int_{-h}^{+h} v_{x} d x$ is the flow rate. 
Its analytical solution exists only for large $K(\geq 5)$ and for small $K(\leq 0.05)$ (Falicki [8], Walicka [15, 16, 37], and Walicki and Walicka [39]):

$$
\begin{gathered}
X_{s}=\frac{3 m}{3 m-1}+[(m+2) K]^{1 / m}, \\
X_{s}=1+[(m+1) K]^{1 /(m+1)} .
\end{gathered}
$$

For intermediate values of $K$ the solutions of (25) may be found only numerical methods. Figures 2 and 3 illustrate the progression of solutions $X_{s}$ versus $K$ for two values of $m$ [8]. Note that for $m=1$, the above values are identical with those obtained by Covey and Stanmore [40].

The functions $S(x)$ and $D(x)$ are given by expressions

$$
\begin{gathered}
S(x)=-\int \tau_{0} \frac{X_{s}}{h} d x \\
D(x)=-\rho \int \frac{1}{R h} \frac{\partial}{\partial x}(R V) d x
\end{gathered}
$$

where

$$
\begin{aligned}
V= & \frac{\tau_{0}^{2 m} h^{3}}{(m+1)^{2} \mu^{2}} X_{s}^{2 m}\left(1-\frac{1}{X_{s}}\right)^{2 m+2} \\
& \times\left[1-\frac{3 m+4}{(m+2)(2 m+3)}\left(1-\frac{1}{X_{s}}\right)\right] .
\end{aligned}
$$

Note that the final form of $S(x)$ and $D(x)$ depend-for $U(x)=$ $U_{o}=$ const - on the values of $K$ and $m$ :

(i) for large values of $K$ and exponent $m$ being a natural number there are

$$
\begin{aligned}
S(x)= & -\alpha\left(\frac{U_{o}}{2}\right)^{\beta} \\
& \times\left[\left(\frac{3 m}{3 m-1}\right) J^{(0 ; \beta)}(x)+\left(A_{m}\right)^{1 / m} J^{(1 / m ; 0)}(x)\right],
\end{aligned}
$$

$$
\begin{aligned}
D(x)= & -\frac{\rho \alpha^{2 m}}{\mu^{2} c_{m}}\left(\frac{U_{o}}{2}\right)^{2 \beta m} \\
& \times\left\{a_{m} \sum_{i=0}^{2 m}(-1)^{i}\left(\frac{3 m}{3 m-1}\right)^{2 m-i} C_{2 m+2}^{i}\right. \\
& \times \sum_{j=0}^{2 m-i}\left(B_{m}\right)^{j / m} C_{2 m-i}^{j} I_{(m)}^{(j ; \beta)}(x) \\
& +b_{m}^{2 m-1}(-1)^{i}\left(\frac{3 m}{3 m-1}\right)^{2 m-1-i} C_{2 m+2}^{i} \\
& \left.\times \sum_{j=0}^{2 m-1-i}\left(B_{m}\right)^{j / m} C_{2 m-1-i}^{j} I_{(m)}^{(j ; \beta)}(x)\right\}
\end{aligned}
$$

where

$$
\begin{gathered}
A_{m}=\frac{(m+2) \mu Q}{2^{2-\beta m} \pi \alpha^{m} U_{o}^{\beta m}}, \quad B_{m}=\left(\frac{3 m-1}{3 m}\right)^{m} A_{m}, \\
a_{m}=2(m+1)^{2}, \\
b_{m}=3 m+4, \quad c_{m}=(m+1)^{2}(m+2)(2 m+3), \\
J^{(\alpha ; \beta)}(x)=\int\left(R^{-\alpha} h^{-(1+2 \alpha)-\beta(1-\alpha m)}\right) d x, \\
C_{\beta}^{\alpha}=\frac{\beta !}{\alpha !(\beta-\alpha) !}, \quad \alpha !=1 \cdot 2 \cdot 3 \cdots \cdots, \beta>\alpha, \\
I_{(m)}^{(j ; \beta)}(x)=\int \frac{1}{R h}\left(R^{1-j / m} h^{3-2 j / m-\beta(2 m-j)}\right)^{\prime} d x .
\end{gathered}
$$

(ii) for large values of $K$ and exponent $m$ being a rational number the formula for $S(x)$ is similar but $D(x)$ takes the form

$$
\begin{aligned}
D(x)= & -\frac{\rho \alpha^{2 m}}{\mu^{2} c_{m}}\left(\frac{U_{o}}{2}\right)^{2 \beta m} \\
& \times\left\{a_{m} \sum_{i=0}^{M_{1}}(-1)^{i} \Gamma_{2 m+2}^{i}\right. \\
& \times \sum_{j=0}^{M_{1}-i} \Gamma_{2 m-i}^{j}\left(\frac{3 m}{3 m-1}\right)^{j}\left(A_{m}\right)^{2-(i+j) / m} J_{(m)}^{(i+j ; \beta)}(x) \\
& +b_{m} \sum_{i=0}^{M_{2}}(-1)^{i} \Gamma_{2 m+2}^{i} \\
& \times \sum_{j=0}^{M_{2}-i} \Gamma_{2 m-1-i}^{j}\left(\frac{3 m}{3 m-1}\right)^{j}\left(A_{m}\right)^{2-(1+i+j) / m} \\
& \left.\times J_{(m)}^{(1+i+j ; \beta)}(x)\right\},
\end{aligned}
$$

where

$$
\begin{gathered}
M_{1}=E(2 m), \quad M_{2}=E(2 m-1), \\
\Gamma_{\alpha}^{i}=\frac{1}{i !} \prod_{k=1}^{i}(\alpha+1-k), \quad \Gamma_{\alpha}^{0}=1, \\
J_{(m)}^{(\alpha ; \beta)}(x)=\int \frac{1}{R h}\left(R^{-1+(\alpha / m)} h^{-1+(2 \alpha / m)-\beta \alpha}\right)^{\prime} d x, \\
\alpha=\left\{\begin{array}{l}
i+j, \\
1+i+j,
\end{array}\right.
\end{gathered}
$$

and $E(\alpha)$ denotes an entire part of $\alpha: \alpha-1<E(\alpha) \leq \alpha$; 
(iii) for small values of $K$ there are

$$
\begin{aligned}
S(x)=- & \alpha\left(\frac{U_{o}}{2}\right)^{\beta} \\
\times & {\left[J^{(0 ; \beta)}(x)+\left(C_{m}\right)^{1 /(m+1)} J^{(1 /(m+1) ; \beta)}(x)\right], } \\
D(x)=- & \frac{2 \rho \alpha^{2 m}}{a_{m} \mu^{2}}\left(\frac{U_{o}}{2}\right)^{\beta m} \\
\times & {\left[\left(C_{m}\right)^{2} J_{(m+1)}^{(0 ; \beta)}(x)\right.} \\
& \quad-\left(2+d_{m}\right)\left(C_{m}\right)^{(2 m+3) /(m+1)} J_{(m+1)}^{(1 ; \beta)}(x) \\
& +3\left(1+d_{m}\right)\left(C_{m}\right)^{2(m+2) /(m+1)} J_{(m+1)}^{(2 ; \beta)}(x) \\
& \left.\quad-6 d_{m}\left(C_{m}\right)^{(2 m+5) /(m+1)} J_{(m+1)}^{(3 ; \beta)}(x)\right],
\end{aligned}
$$

where

$$
\begin{gathered}
C_{m}=\frac{(m+1) \mu Q}{2^{2-\beta m} \pi \alpha^{m} U_{o}^{\beta m}}, \\
d_{m}=\frac{3 m+4}{(m+2)(2 m+3)},
\end{gathered}
$$

$$
\begin{aligned}
& J_{(m+1)}^{(j ; \beta)}(x) \\
& \quad=\int \frac{1}{R h}\left(R^{-(m+1+j) /(m+1)} h^{-(m+1+2 j) /(m+1)-2 \beta m}\right)^{\prime} d x .
\end{aligned}
$$

For $\tau_{0}(x)=\tau_{0}=$ const (the coefficient $\beta=0$ but $\alpha=\tau_{0}$ ) there are [8]

(iv) for large values of $K$ and exponent $m$ being a natural number:

$$
S(x)=-\tau_{o}\left[\left(\frac{3 m}{3 m-1}\right) J^{(0)}(x)+\left(A_{m}\right)^{1 / m} J^{(1 / m)}(x)\right],
$$

$$
\begin{aligned}
D(x)= & -\frac{\rho \tau_{0}^{2 m}}{\mu^{2} c_{m}} \\
& \times\left\{a_{m} \sum_{i=0}^{2 m}(-1)^{i}\left(\frac{3 m}{3 m-1}\right)^{2 m-i} C_{2 m+2}^{i}\right. \\
& \times \sum_{j=0}^{2 m-i}\left(B_{m}\right)^{j / m} C_{2 m-i}^{j} I_{(m)}^{(j)} \\
& +b_{m} \sum_{i=0}^{2 m-1}(-1)^{i}\left(\frac{3 m}{3 m-1}\right)^{2 m-1-i} C_{2 m+2}^{i} \\
& \left.\times \sum_{j=0}^{2 m-1-i}\left(B_{m}\right)^{j / m} C_{2 m-1-i}^{j} I_{(m)}^{(j)}\right\},
\end{aligned}
$$

where

$$
\begin{gathered}
A_{m}=\frac{(m+2) \mu Q}{4 \pi \tau_{0}^{m}}, \quad J^{(\alpha)}(x)=J^{(\alpha ; 0)}(x), \\
I_{(m)}^{(j)}(x)=I_{(m)}^{(j ; 0)}(x) ;
\end{gathered}
$$

(v) for large values of $K$ and exponent $m$ being a rational number the formula for $S(x)$ is similar and $D(x)$ takes the form

$$
\begin{aligned}
D(x)= & -\frac{\rho \tau_{0}^{2 m}}{\mu^{2} c_{m}} \\
& \times\left\{a_{m} \sum_{i=0}^{M_{1}}(-1)^{i} \Gamma_{2 m+2}^{i}\right.
\end{aligned}
$$

$$
\left.\begin{array}{c}
\times \sum_{j=0}^{M_{1}-i} \Gamma_{2 m-i}^{j}\left(\frac{3 m}{3 m-1}\right)^{j}\left(A_{m}\right)^{2-(i+j) / m} J_{(m)}^{(i+j)} \\
+b_{m} \sum_{i=0}^{M_{2}}(-1)^{i} \Gamma_{2 m+2}^{i} \\
\times \sum_{j=0}^{M_{2}-i} \Gamma_{2 m-1-i}^{j}\left(\frac{3 m}{3 m-1}\right)^{j}\left(A_{m}\right)^{2-(1+i+j) / m} \\
\times J_{(m)}^{(1+i+j)}
\end{array}\right\},
$$

where

$$
J_{(m)}^{(\alpha)}(x)=J_{(m)}^{(\alpha ; 0)}(x)
$$

(vi) for small values of $K$ there are

$$
\begin{aligned}
S(x)=- & \tau_{0}\left[J^{(0)}(x)+\left(C_{m}\right)^{1 /(m+1)} J^{(1 /(m+1))}(x)\right], \\
D(x)=- & \frac{2 \rho \tau_{0}^{2 m}}{a_{m} \mu^{2}} \\
\times & {\left[\left(C_{m}\right)^{2} A^{(1)}(x)\right.} \\
& -\left(2+d_{m}\right)\left(C_{m}\right)^{(2 m+3) /(m+1)} J_{(m+1)}^{(1)}(x) \\
& +3\left(1+d_{m}\right)\left(C_{m}\right)^{(2(m+2)) /(m+1)} J_{(m+1)}^{(2)}(x) \\
& \left.-6 d_{m}\left(C_{m}\right)^{(2 m+5) /(m+1)} J_{(m+1)}^{(3)}(x)\right],
\end{aligned}
$$


where

$$
\begin{gathered}
A^{(1)}(x)=A^{(1 ; 0)}(x), \quad J^{(0)}(x)=J^{(0 ; 0)}(x), \\
J^{(1 /(m+1))}(x)=J^{(1 /(m+1) ; 0)}(x), \\
J_{(m)}^{(\alpha)}(x)=J_{(m)}^{(\alpha ; 0)}(x), \quad J_{(m+1)}^{(j)}(x)=J_{(m+1)}^{(j ; 0)}(x) .
\end{gathered}
$$

The prim denotes the differentiation with respect to $x$.

\section{Examples of Flow and Graphic Presentation of Some Results}

Taking into account the results obtained in the previous section we will present the pressure distribution in the clearance of constant thickness between two parallel disks as shown in Figure 4; to this aim introduce the following dimensionless parameters:

$$
\begin{gathered}
\tilde{x}=\frac{x}{x_{o}}, \quad \widetilde{R}=\frac{R}{R_{o}}=\tilde{x}, \\
\widetilde{p}=\frac{\rho\left(p-p_{o}\right)(2 h)^{4 / m} Q^{2-2 / m}}{(2 \pi)^{2-2 / m} \mu^{2 / m} R_{o}^{4-2 / m} R_{\lambda}}, \\
R_{\lambda}=\frac{\rho Q^{2-1 / m}}{(2 \pi)^{2-1 / m} \mu^{1 / m} R_{o}^{3-1 / m}(2 h)^{1-2 / m}}, \\
K_{\mathrm{SV}}=\frac{2^{\beta m-2} \mu Q h^{\beta m-2}}{\pi \alpha^{m} R_{o} U_{o}^{\beta m}},
\end{gathered}
$$

if $\beta=0$, then $\alpha=\tau_{0}=$ const and

$$
K_{\mathrm{SV}}=\frac{\mu \mathrm{Q}}{4 \pi R_{o} h^{2} \tau_{0}^{m}}
$$

here $R_{\lambda}$ is the modified Reynolds number, and $K_{\mathrm{SV}}$ is the ER plasticity number (de Saint-Venant number). Note that large values of the ER plasticity number correspond to the flow with small core ( $\left.\operatorname{small} h_{0} / h\right)$.

Nondimensional formula for pressure distribution in ER flow of the Herschel-Bulkley fluid has the form

$$
\widetilde{p}=\widetilde{S}(\tilde{x})-\widetilde{S}_{o}+\widetilde{D}(\widetilde{x})-\widetilde{D}_{o} ;
$$

this formula may be used to model the pressure distribution in a footstep bearing.

Note that for $m=1$, all the above formulae represent the Bingham ERF flow. The nondimensional pressure distributions for the Herschel-Bulkley ERF flow in a clearance between two parallel disks are presented in Figures 5, 6, 7, 8, and 9. The values of nondimensional parameters such as $R_{\lambda}$, $K_{\mathrm{SV}}$, and $m$, are defined on the experimental data taken from the measurements presented in $[8,12]$.

Let us consider now the pressure distribution in the clearance of constant thickness between two concentric

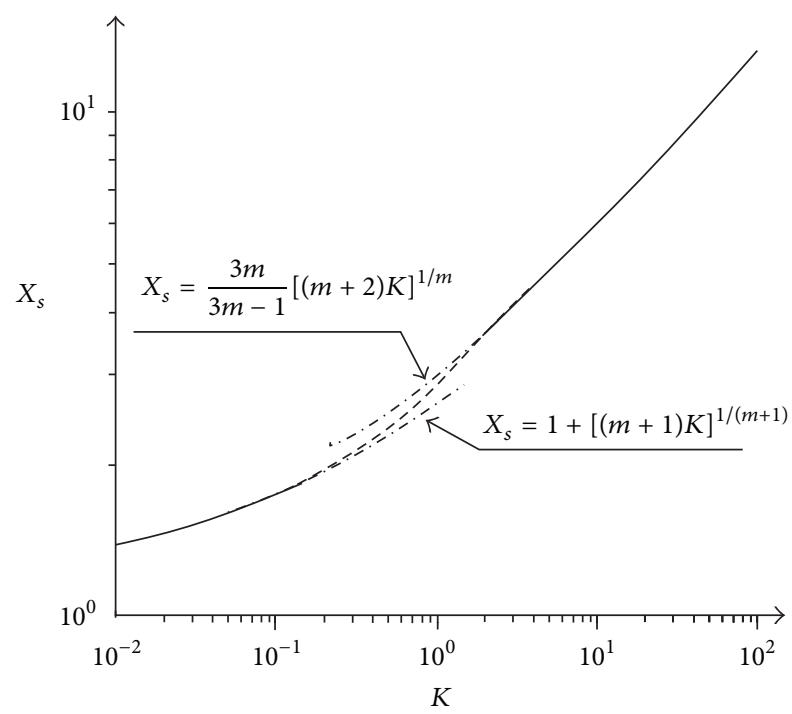

FIgURE 2: Curves illustrating the progression of $X_{s}$ versus $K$ for $m=$ 2.5; continuous lines determine the exact solution.

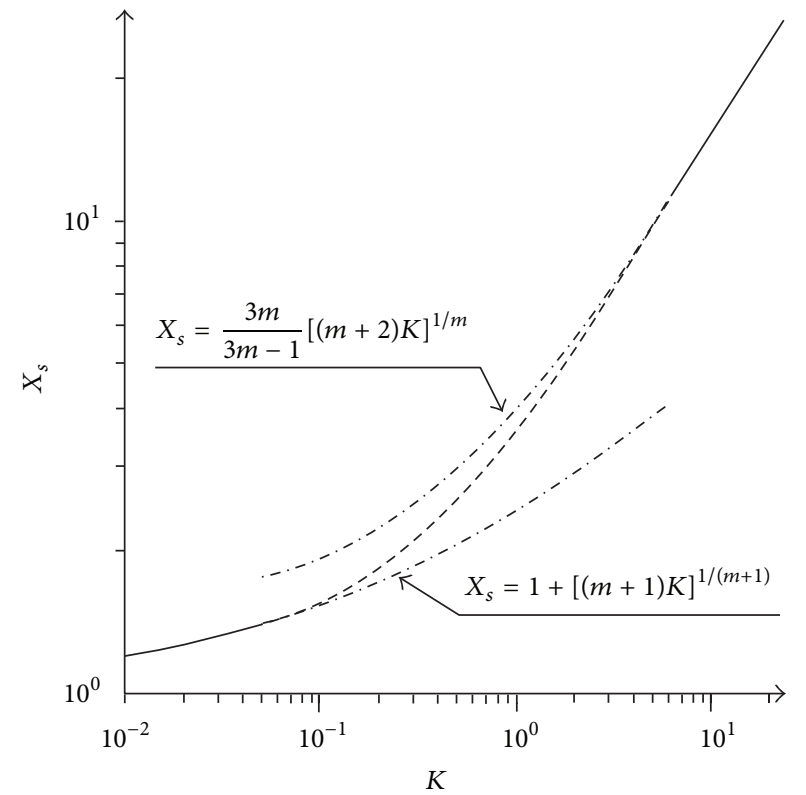

FIgURE 3: Curves illustrating the progression of $X_{s}$ versus $K$ for $m=$ $4 / 3$; continuous lines determine the exact solution.

spheres surfaces shown in Figure 10. To this aim introduce the following dimensionless parameters:

$$
\begin{gathered}
\tilde{x}=\frac{x}{R_{s}}=\varphi, \quad \widetilde{R}=\frac{R}{R_{s}}=\sin \varphi, \\
\widetilde{p}=\frac{\rho\left(p-p_{o}\right)(2 h)^{4 / m} Q^{2-2 / m}}{(2 \pi)^{2-2 / m} \mu^{2 / m} R_{s}^{4-2 / m} R_{\lambda}}, \\
R_{\lambda}=\frac{\rho Q^{2-1 / m}}{(2 \pi)^{2-1 / m} \mu^{1 / m} R_{s}^{3-1 / m}(2 h)^{1-2 / m}}, \\
K_{\mathrm{SV}}=\frac{2^{\beta m-2} \mu \mathrm{Qh}^{\beta m-2}}{\pi \alpha^{m} R_{s} U_{o}^{\beta m}} .
\end{gathered}
$$




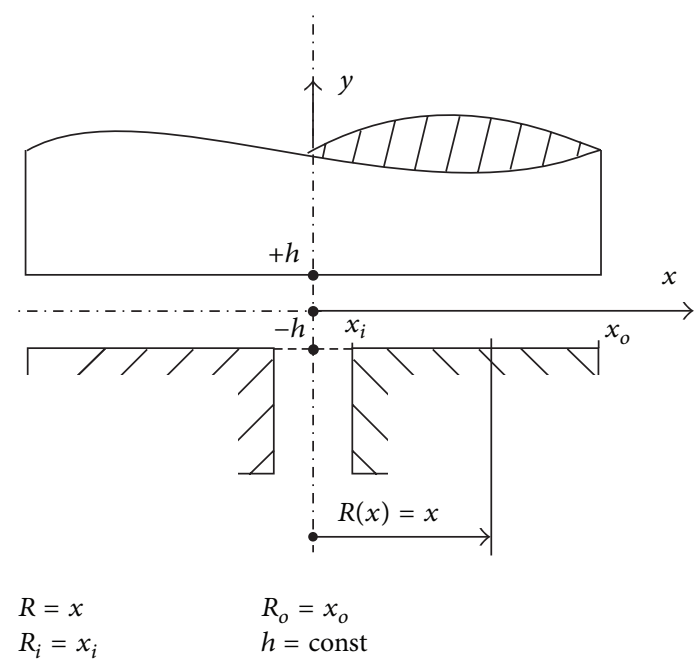

FIgURE 4: Clearance between two parallel disks.

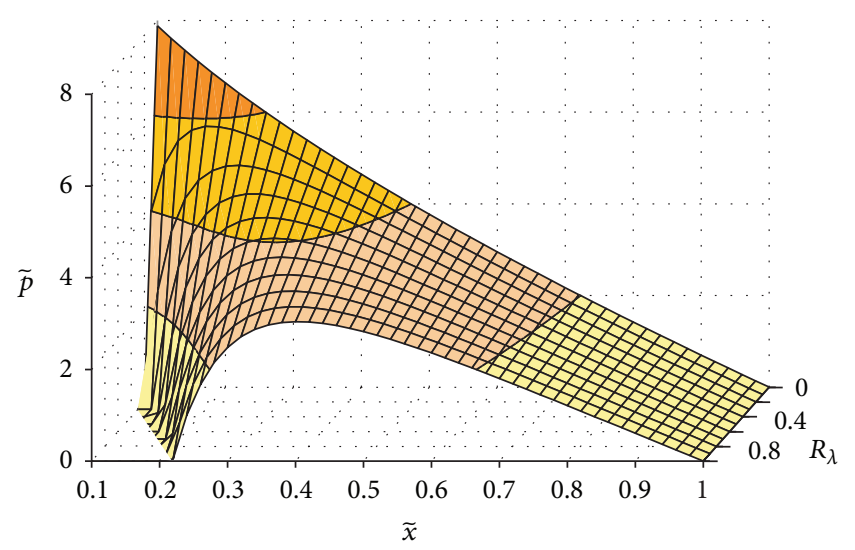

FIgURE 5: Pressure distributions $\tilde{p}$ in the clearance between parallel disks for different values of $R_{\lambda}$, large value of $K_{\mathrm{SV}}=5$, and exponent $2 m(m=2.5)$ being a natural number.

Note that if $\beta=0$ then $\alpha=\tau_{0}=$ const and $K_{\mathrm{SV}}=$ $\mu \mathrm{Q} / 4 \pi R_{s} h^{2} \tau_{0}^{m}$. The nondimensional pressure distributions are also given by formula (41); at present it models the pressure distribution in a spherical bearing.

The pressure distributions for the Herschel-Bulkley ERF flow in a clearance between concentric spherical surfaces are presented in Figures 11, 12, 13, and 14.

\section{Conclusions}

From the general considerations, formulae and graphs presented here for the Herschel-Bulkley ERF flows in the narrow clearance of constant thickness between parallel disks and concentric spherical surfaces shown in Figures 4 and 10 one may conclude that the pressure values

(i) decrease with the increase of the modified Reynolds number $R_{\lambda}$,

(ii) increase with the decrease of the nonlinearity index $m$,

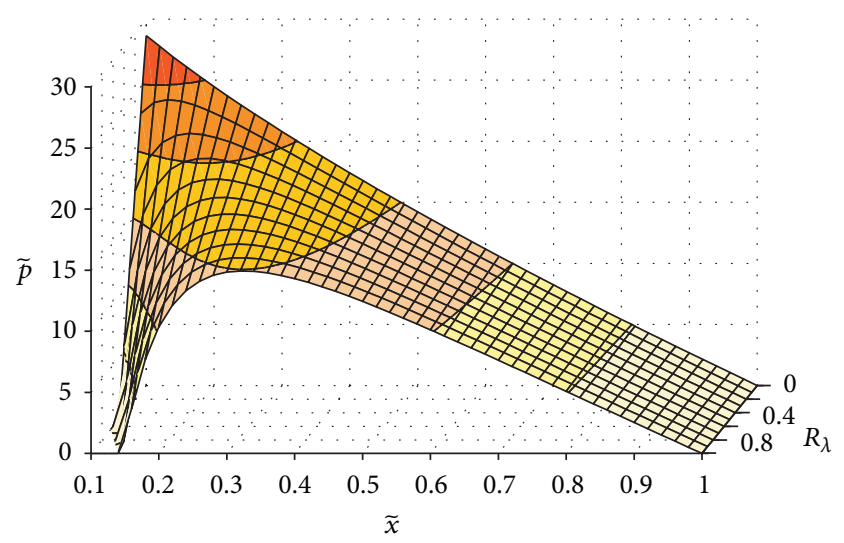

Figure 6: Pressure distributions $\tilde{p}$ in the clearance between parallel disks for different values of $R_{\lambda}$, large value of $K_{\mathrm{SV}}=5$, and exponent $2 m(m=4 / 3)$ being a rational number.

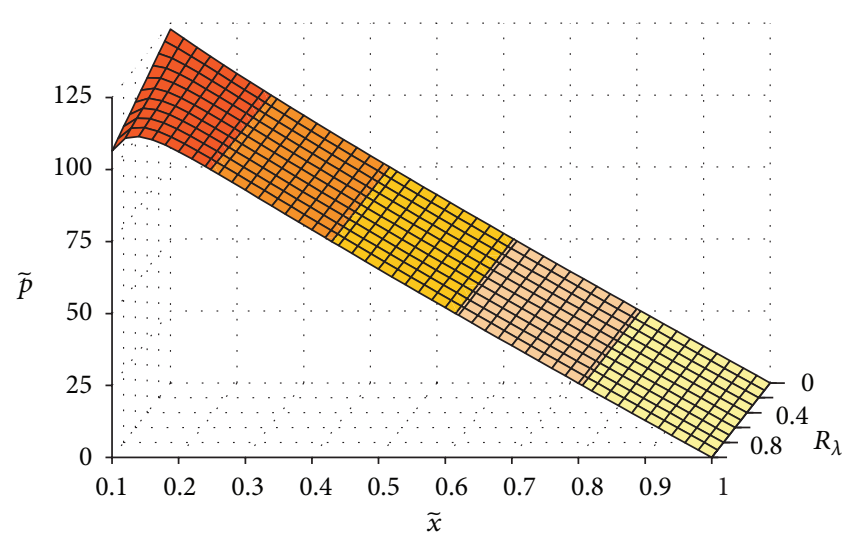

Figure 7: Pressure distributions $\widetilde{p}$ in the clearance between parallel disks for different values of $R_{\lambda}$, small value of $K_{\mathrm{SV}}=0.01$, and exponent $2 m(m=4 / 3)$ being a rational number.

(iii) increase with the decrease of the de Saint-Venant ER number $K_{\mathrm{SV}}$,

(iv) are larger between concentric spherical surfaces than these ones between parallel disks for $2 m$ being natural numbers and large values of $K_{\mathrm{SV}}$,

(v) for $m$ being rational numbers it is inversely.

For small values of the de Saint-Venant ER number $K_{S V}$ the influence of the Reynolds number $R_{\lambda}$ on the pressure values is inconsiderable. Therefore, for these values of $K_{S V}$ the Herschel-Bulkley ERF flows may be considered without inertia effects. It is seen from the results obtained here, that the pressure values are larger for the flows between concentric spherical surfaces than these values for the flows between parallel disks. 


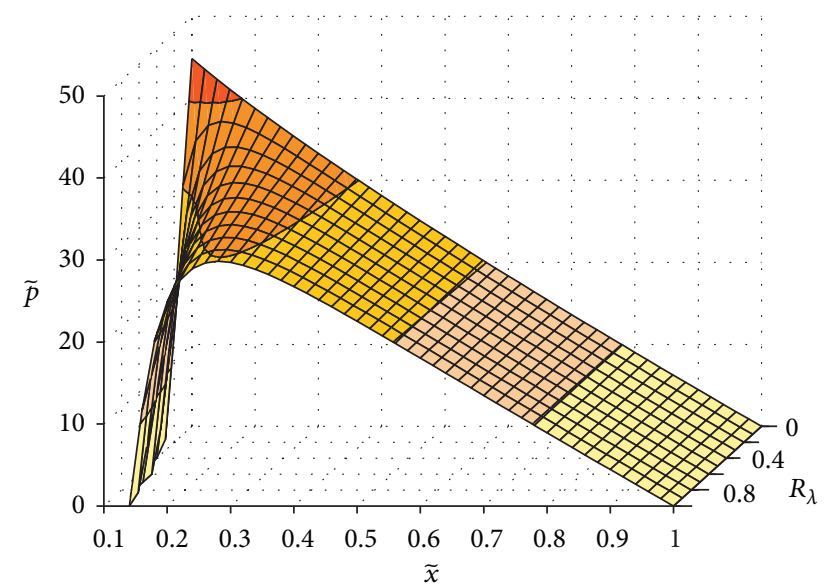

FIGURE 8: Pressure distributions $\tilde{p}$ in the clearance between parallel disks for different values of $R_{\lambda}$, small value of $K_{\mathrm{SV}}=0.05$, and exponent $2 m(m=4 / 3)$ being a rational number.

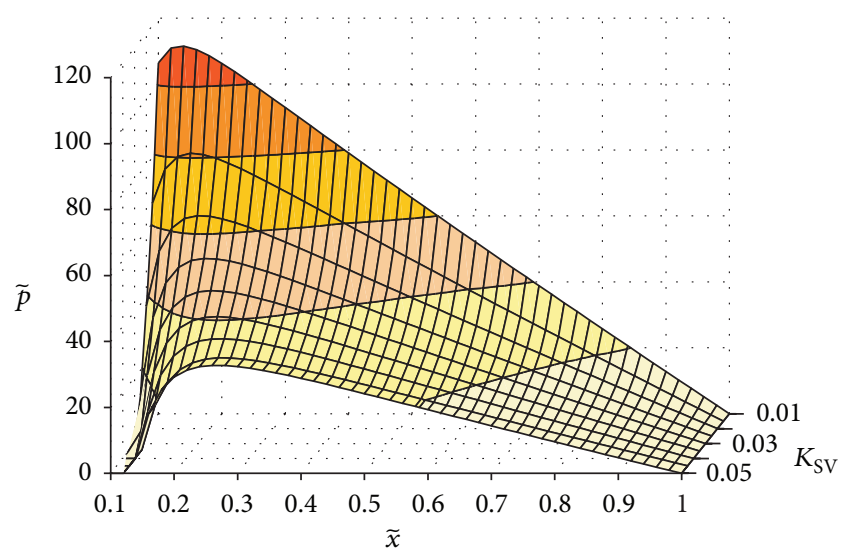

Figure 9: Pressure distributions $\widetilde{p}$ in the clearance between two parallel disks for constant value of $R_{\lambda}(=1)$, different values of $K_{\mathrm{SV}}$, and exponent $2 m(m=4 / 3)$ being a rational number.

Generally, it may be concluded that for the HerschelBulkley ERF flows in the clearance between two surfaces of revolution the pressure values

(i) are larger for the flow in the clearances with curvilinear generating lines than these ones for the flow in clearances with rectilinear generating lines for the nonlinearity index $2 m$ being natural numbers; for $m$ being rational numbers this phenomenon progresses inversely.

Note that the results obtained here for $\tau_{0}=$ const and $m$ being natural numbers are similar to those of the work by Walicki and Walicka [39].

The formulae for the pressure distribution obtained previously may be used to model its distribution in slide thrust bearings-of arbitrary curvilinear shapes-lubricated by ER lubricants.

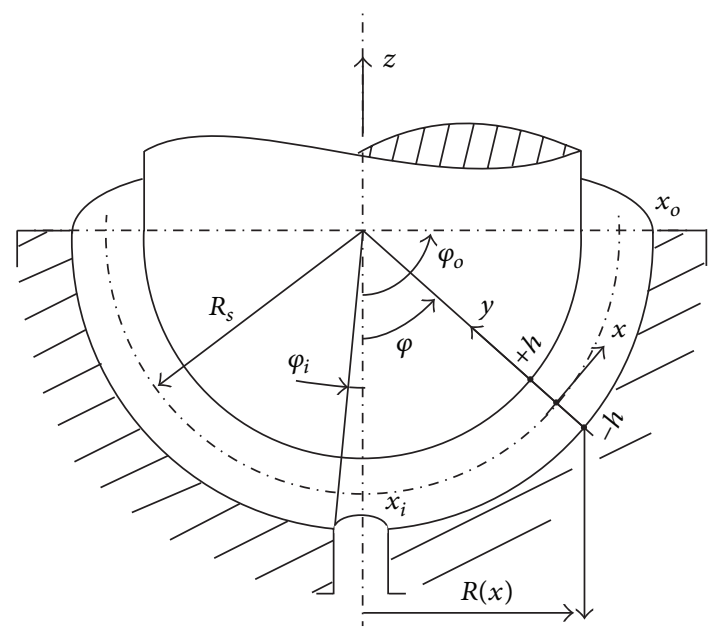

$$
\begin{aligned}
R & =R_{s} \sin \varphi & & R_{o}=R_{s} \sin \varphi_{o} \\
\varphi & =\frac{x}{R_{s}} & & h=\text { const } \\
R_{i} & =R_{s} \sin \varphi_{i} & &
\end{aligned}
$$

FIGURE 10: Clearance between concentric spherical surfaces.

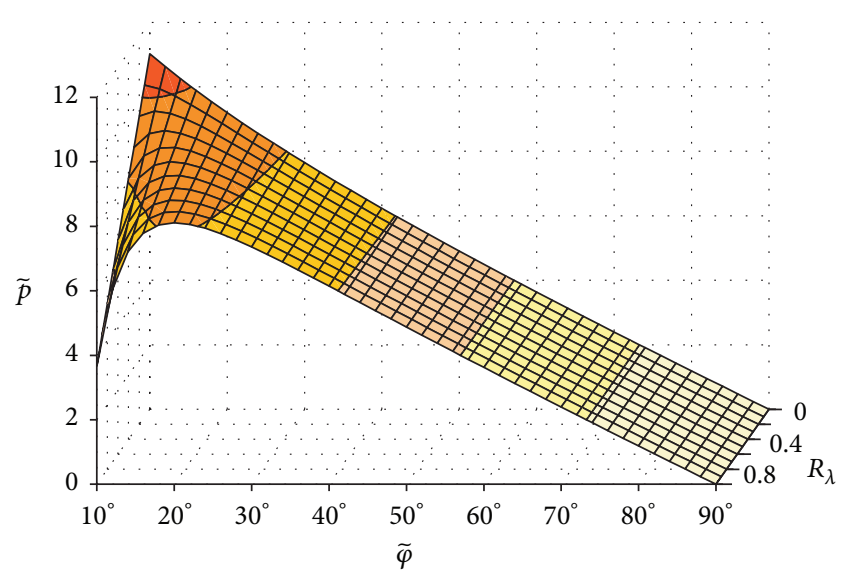

Figure 11: Pressure distributions $\widetilde{p}$ in the clearance between concentric spherical surfaces for different values of $R_{\lambda}$, large value of $K_{\mathrm{SV}}=5$, and exponent $2 m(m=2.5)$ being a natural number.

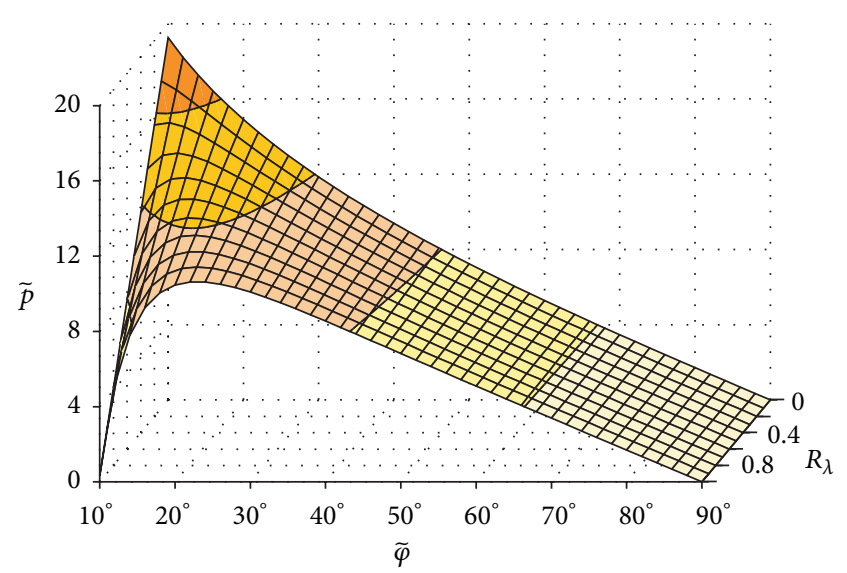

FIgURE 12: Pressure distributions $\widetilde{p}$ in the clearance between concentric spherical surfaces for different values of $R_{\lambda}$, large value of $K_{\mathrm{SV}}=5$, and exponent $2 m(m=4 / 3)$ being a rational number. 


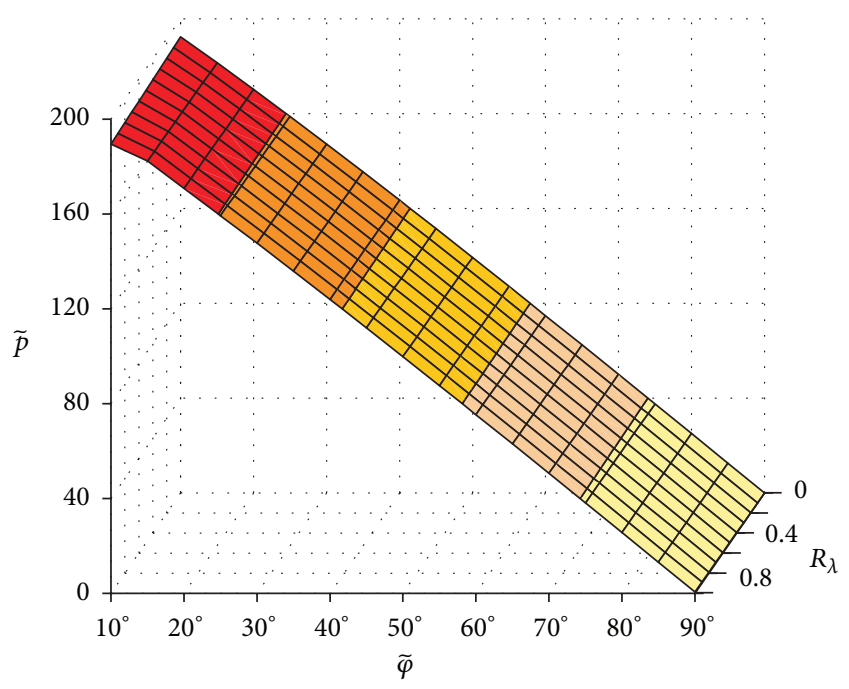

FIgURE 13: Pressure distributions $\widetilde{p}$ in the clearance between concentric spherical surfaces for different values of $R_{\lambda}$, small value of $K_{S V}=0.01$, and exponent $2 m(m=4 / 3)$ being a rational number.

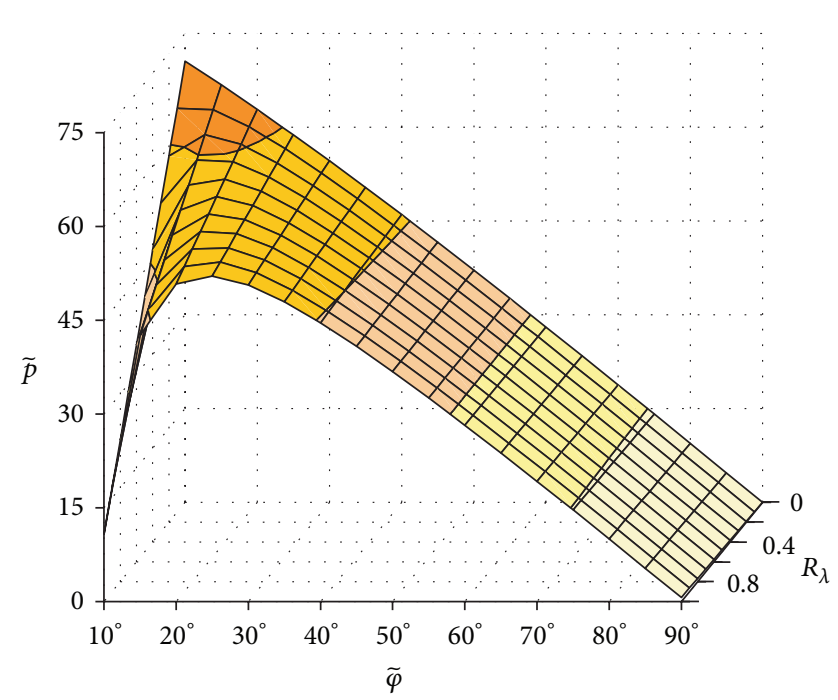

FIgURE 14: Pressure distributions $\widetilde{p}$ in the clearance between concentric spherical surfaces for different values of $R_{\lambda}$, small value of $K_{\mathrm{SV}}=0.05$ and exponent $2 m(m=4 / 3)$ being a rational number.

\section{Nomenclature}

$A_{m}$ : $\quad$ Auxiliary function defined by formulae $(30)_{1}$ or $(36)_{1}$

$B_{m}$ : $\quad$ Auxiliary function defined by formula $(30)_{2}$

$D(x)$ : Auxiliary function defined by formula $(28)_{2}$ or $(29 a),(29 b),(31 a)$, and (31b) or $(34 \mathrm{a})$ and $(34 \mathrm{~b})$, respectively

$h: \quad$ Half of the fluid film thickness

$I_{(m)}^{(j ; \beta)}$ : Auxiliary function defined by formula $(30)_{8}$

$J^{(\alpha ; \beta)}(x)$ : Auxiliary function defined by formula $(30)_{6}$
$J_{(m)}^{(j ; \beta)}(x)$ : Auxiliary function defined by formula $(35)_{3}$

$K: \quad$ Plasticity number

$K_{\mathrm{SV}}$ : De Saint-Venant plasticity number

$m: \quad$ Power-law index

$p: \quad$ Pressure

$Q: \quad$ Flow rate

$R, R(x)$ : Local radius

$R_{i}$ : $\quad$ Inlet radius to the bearing clearance

$R_{o}$ : Outlet radius from the bearing clearance

$R_{\lambda}$ : $\quad$ Modified Reynolds number

$S: \quad$ Signum function

$S(x)$ : Auxiliary function defined by formulae $(28)_{1},(28 a)$, and (28b) or (33a) and (33b), respectively

$T_{y x}$ : Component of the shear stress tensor

$U$ : $\quad$ Applied voltage

$v_{x}, v_{y}: \quad$ Velocity components

$\alpha, \beta: \quad$ ER experimental constants

$\rho: \quad$ Fluid density.

$\varphi$ : $\quad$ Central angle of spherical surface

$\tau: \quad$ Shear stress

$\tau_{0}: \quad$ Yield shear stress.

\section{References}

[1] H. A. Barnes, J. F. Hutton, and K. Walters, An Introduction to Rheology, Elsevier, Amsterdam, The Netherlands, 1989.

[2] J. S. Basavaraja, S. C. Sharma, and S. C. Jain, "A study of misaligned electrorheological fluid lubricated hole-entry hybrid journal bearing," Tribology International, vol. 43, no. 5-6, pp. 1059-1064, 2010.

[3] R. B. Bird, R. C. Armstrong, and O. Hassager, Dynamic of Polymeric Liquids, vol. 1, John Wiley, New York, NY, USA, 1977.

[4] R. B. Bird, C. F. Curtis, R. C. Armstrong, and O. Hassager, Dynamic of Polymeric Liquids, vol. 2, John Wiley, New York, NY, USA, 1987.

[5] N. Casson, Rheology of Dispersed Systems, C.C. Mills, New York, NY, USA, 1959.

[6] C. D. Han, Rheology and Processing of Polymeric Materials, Oxford University Press, 2009.

[7] R. P. Chhabra and J. F. Richardson, Non-Newtonian Flow and Applied Rheology, Butterworth-Heinemann, Oxford, UK, 2008.

[8] J. Falicki, The influence viscoplastic lubricants on the pressure distributions in the thrust slide bearings [Ph.D. thesis], University Press, Zielona Góra, Poland, 2007.

[9] J. Falicki and A. Walicka, "Effect of the choice of oil additives on rheological properties of engine oils," International Journal Applied Mechanics and Engineering, vol. 10, pp. 309-315, 2005.

[10] N. Heinrichson, On the design of tilting-pad thrusts bearings [Ph.D. thesis], Lyngby, Denmark, 2006.

[11] R. I. Tanner, Engineering Rheology, Oxford University Press, 2000.

[12] N. Roussel, Analyse des écoulements des fluides homogènes complexes et plastiques diphasiques: application à l'essai de compression simple [Ph.D. thesis], Rennes, France, 2001.

[13] E. Walicki, Rheodynamics of Slide Bearings Lubrication, University Press, Zielona Góra, Poland, 2005 (in Polish). 
[14] A. Walicka, "Pressure distribution in a squeeze film of a Shulman fluid between surfaces of revolution," International Journal of Engineering Science, vol. 69, pp. 33-48, 2013.

[15] A. Walicka, Rheodynamics of Non-Newtonian Fluids Flow in Straight and Curved Channels, University Press, Zielona Góra, Poland, 2002 (in Polish).

[16] A. Walicka, Rotational Flows of the Rheologically Complex Media in Thin Annular Channels, University Press, Zielona Góra, Poland, 2002 (in Russian).

[17] W. M. Winslow, "Induced fibration of suspensions," Journal of Applied Physics, vol. 20, no. 12, pp. 1137-1140, 1949.

[18] S. Y. Jung and S.-B. Choi, "Analysis of a short squeeze-film damper operating with electrorheological fluids," Tribology Transactions, vol. 38, no. 4, pp. 857-862, 1995.

[19] K. Kobayashi, K. Okamura, T. Sakai, and M. Sato, "Evaluation of the flow rate of an electro-rheological fluid (Corn StarchKerosene) flowing through a narrow channel formed by a pair of electrodes," Canadian Journal of Chemical Engineering, vol. 74, no. 3, pp. 394-398, 1996.

[20] M. V. Korobko, Electrostructured (Electrorheological) Fluids: Dynamics Singularities and Possibility of Aplication, BAS Press, Minsk, Russia, 1996.

[21] Z. P. Shulman, R. G. Gorodkin, E. V. Korobko, and V. K. Gleb, "The electrorheological effect and its possible uses," Journal of Non-Newtonian Fluid Mechanics, vol. 8, no. 1-2, pp. 29-41, 1981.

[22] Z. P. Shulman and V. Nosov, "Rotation of the axisimmetric dielectric bodies (DEB) in electrorheological suspensions (ERS)," International Journal of Modern Physics B, vol. 10, no. 23-24, pp. 2903-2915, 1996.

[23] D.-Y. Lee and N. M. Wereley, "Quasi-steady Herschel-Bulkley analysis of electro- and magneto-rheological flow mode dampers," Journal of Intelligent Material Systems and Structures, vol. 10, no. 10, pp. 761-769, 1999.

[24] Y. T. Choi, J. U. Cho, S. B. Choi, and N. M. Wereley, "Constitutive models of electrorheological and magnetorheological fluids using viscometers," Smart Materials and Structures, vol. 14, no. 5, pp. 1025-1036, 2005.

[25] A. Walicka, J. Falicki, and E. Walicki, "Flows of electro- and magneto-rheological fluids in curved clearances," in RheologyTheory and Application, M. Dziubiński and K. Antosik, Eds., pp. 369-398, Warsaw, Poland, 2011.

[26] A. D. Dimarogonas and A. Kollias, "Electrorheological fluidcontrolled "smart" journal bearings", Tribology Transactions, vol. 35, no. 4, pp. 611-618, 1992.

[27] J. Peng and K.-Q. Zhu, "Effects of electric field on hydrodynamic characteristics of finite-length ER journal bearings," Tribology International, vol. 39, no. 6, pp. 533-540, 2006.

[28] A. K. El Wahed, J. L. Sproston, R. Stanway, and E. W. Williams, "An improved model of ER fluids in squeeze-flow through model updating of the estimated yield stress," Journal of Sound and Vibration, vol. 268, no. 3, pp. 581-599, 2003.

[29] S. Lim, S.-M. Park, and K.-I. Kim, "AI vibration control of highspeed rotor systems using electrorheological fluid," Journal of Sound and Vibration, vol. 284, no. 3-5, pp. 685-703, 2005.

[30] Z. P. Shulman, Convective Heat Transfer of Rheologically Complex Fluids, Energy, Moscow, Russia, 1975 (in Russian).

[31] W. H. Herschel, "Viscosity and friction," SAE Journal, vol. 10, pp. 31-38, 1922.

[32] E. Walicki, A. Walicka, and J. Falicki, "Inertia effects of viscoplastic lubricant in curved squeeze film," Applied Mechanics and Engineering, vol. 4, pp. 99-108, 1999.
[33] K. P. Vishwanath and A. Kandasamy, "Inertia effects in circular squeeze film bearing using Herschel-Bulkley lubricants," Applied Mathematical Modelling, vol. 34, no. 1, pp. 219-227, 2010.

[34] T. C. Jordan and M. T. Shaw, "Electrorheology," IEEE Transactions on Electrical Insulation, vol. 24, no. 5, pp. 849-878, 1989.

[35] Y. Otsubo, "Electrorheological properties of silica suspensions," Journal of Rheology, vol. 36, no. 3, pp. 479-496, 1992.

[36] M. Whittle, R. Firoozian, D. J. Peel, and W. A. Bullough, "Decomposition of the pressure response in an ER valve control system," Journal of Intelligent Material Systems and Structures, vol. 5, no. 1, pp. 105-111, 1994.

[37] A. Walicka, "Analysis of polymers flow through dies of forming devices," Applied Mechanics and Engineering, vol. 4, no. 2, pp. 341-361, 1999.

[38] A. Walicka and J. Falicki, "Pressure distributions in a curvilinear thrust hydrostatic bearing lubricated by a Herschel-Bulkley fluid," International Journal of Applied Mechanics and Engineering, vol. 13, no. 2, pp. 543-552, 2008.

[39] E. Walicki and A. Walicka, "Reynolds number effects in the flow of an electrorheological fluid between fixed surfaces of revolution," Journal of Intelligent Material Systems and Structures, vol. 9, no. 8, pp. 662-666, 1998.

[40] G. H. Covey and B. R. Stanmore, "Use of the parallel-plate plastometer for the characterisation of viscous fluids with a yield stress," Journal of Non-Newtonian Fluid Mechanics, vol. 8, no. 34, pp. 249-260, 1981. 

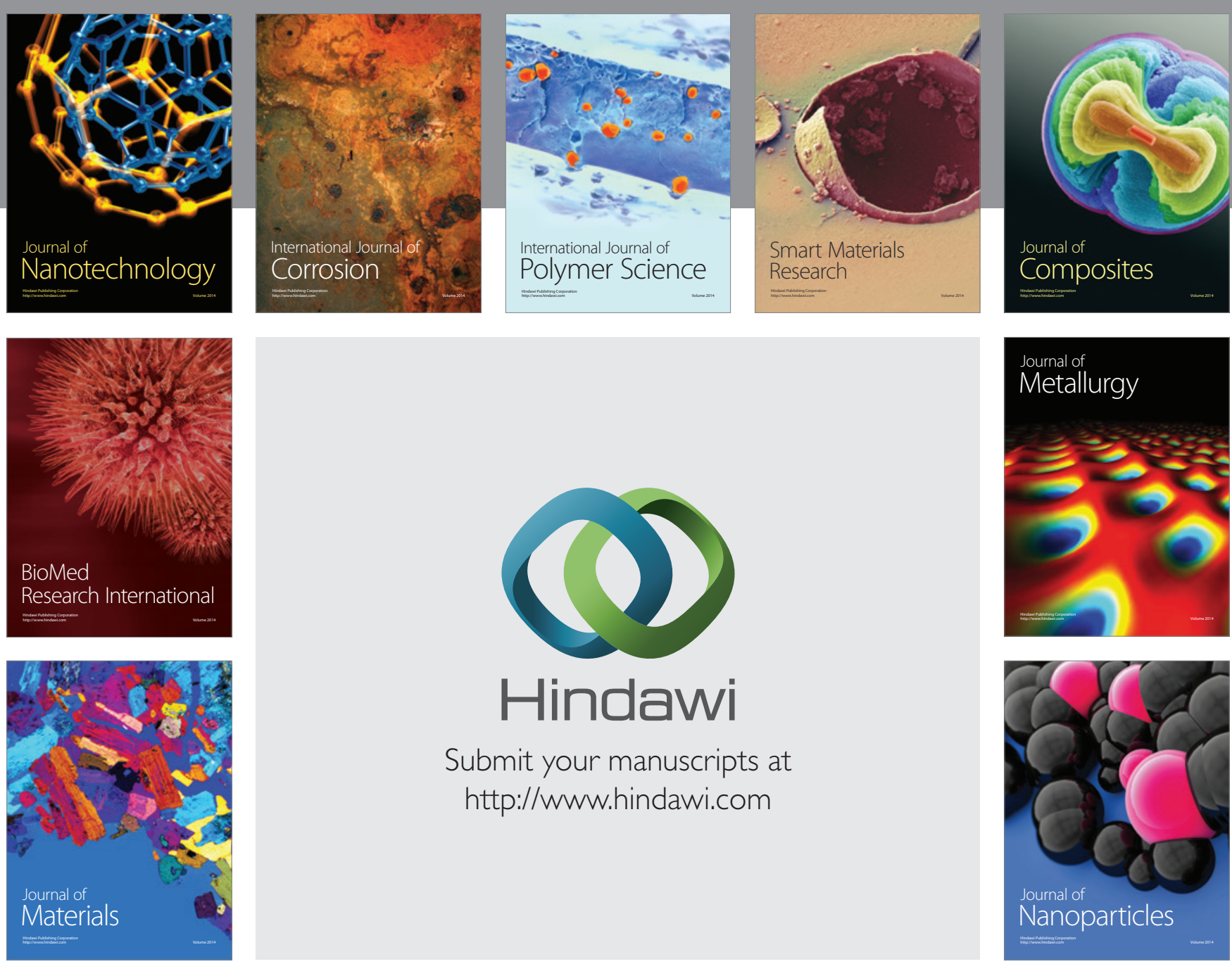

Submit your manuscripts at http://www.hindawi.com
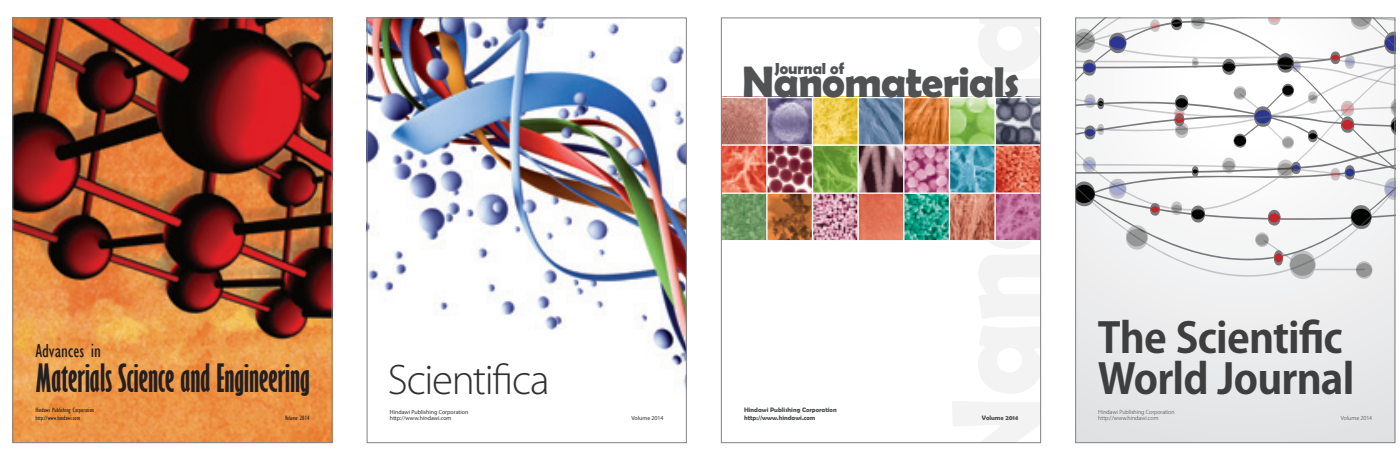

\section{The Scientific World Journal}
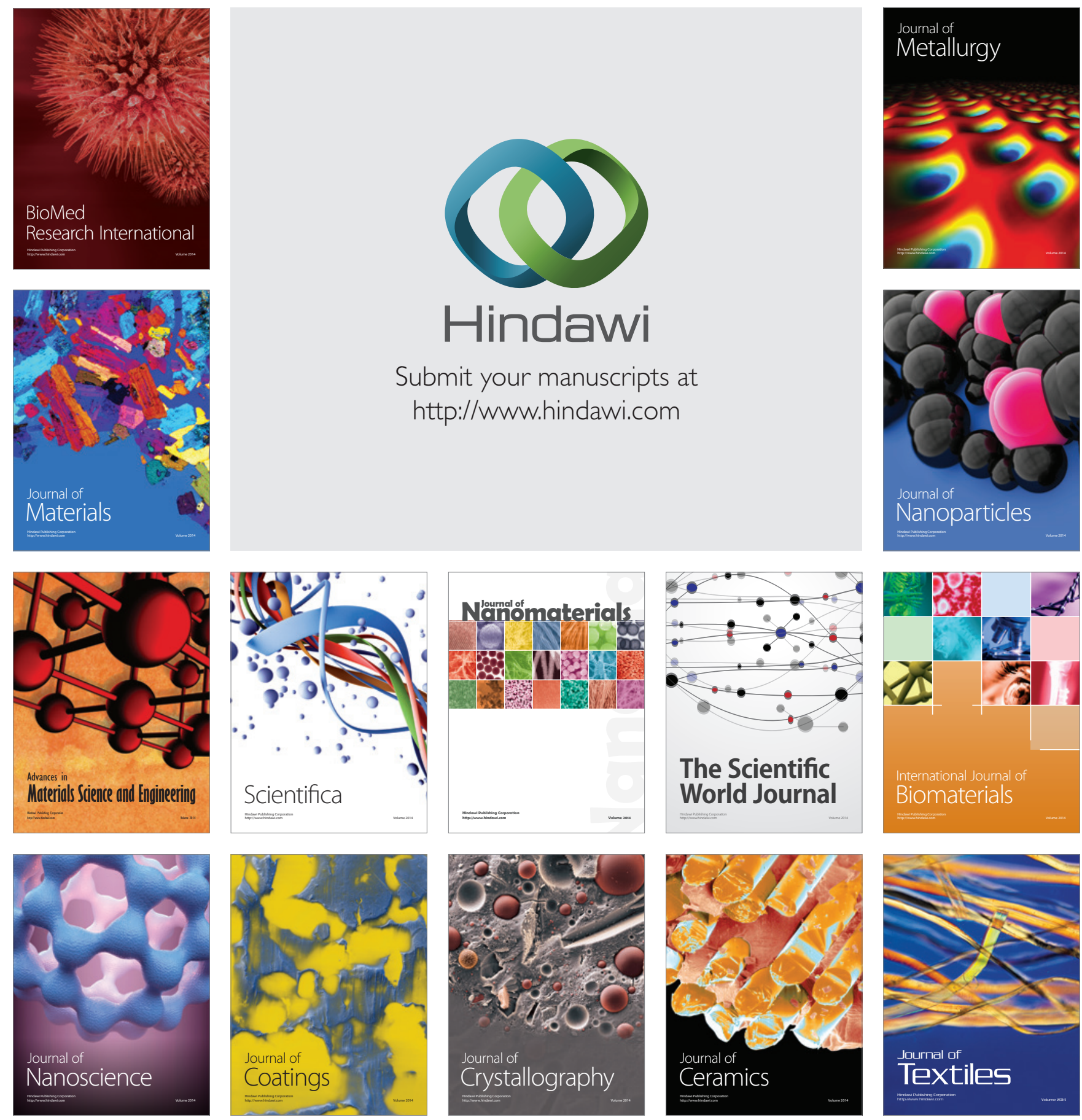